and 48 weeks. In addition, 58 patients underwent radiographic scoring (using the Sharp-van der Heijde scale) at baseline and 48 weeks.

Mean hand BMD at baseline was $0.43 \mathrm{~g} / \mathrm{cm}^{2}$; the mean reduction of hand BMD from baseline at 24 weeks and 48 weeks was $2.5 \%$ and $2.6 \%$, respectively. Hand bone loss exceeding the smallest detectable change (SDC) was present in $46.8 \%$ and $58.2 \%$ of patients at 24 weeks and 48 weeks, respectively. Of the 58 patients who underwent radiographic evaluation, $22 \%$ had radiographic joint damage progression exceeding the SDC value at 48 weeks, compared with $57 \%$ who had BMD loss exceeding the SDC value at 48 weeks.

The authors conclude that hand BMD loss, measured by DEXA, is a more sensitive outcome measure than radiographic bone damage scores in patients with early RA, and should be employed in clinical studies, particularly those that investigate the effects of potent treatments.

Original article Haugeberg G et al. (2007) Hand bone densitometry: a more sensitive standard for the assessment of early bone damage in rheumatoid arthritis. Ann Rheum Dis 66: 1513-1517

\section{Presence of bone edema on MRI might determine disease severity in patients with early RA}

The scoring systems used to evaluate MRI findings in patients with rheumatoid arthritis (RA), such as the Outcome Measures in Rheumatology RA MRI studies (OMERACTRAMRIS), are complex. Tamai and colleagues, therefore, evaluated MRI findings in patients with early-stage RA, using images of wrists and fingers only.

The study included 80 patients with RA from a single Japanese center. Median disease duration at study entry was 3 months. MRI was performed on both wrists and hands; the images were evaluated for bone edema, bone erosion and synovitis at 15 wrist and finger joints on each hand. Serologic tests were also performed, including measurement of matrix metalloproteinase (MMP)3, C-reactive protein (CRP), anti-cyclic citrullinated peptide (anti-CCP) antibody and interleukin (IL)-6 levels.

The number of edema-positive bones correlated with the number of bones scored positive for synovitis and erosion on MRI, and also with serum levels of anti-CCP antibodies, CRP, MMP3 and IL-6. There was also a positive correlation between the 28-joint disease activity score-CRP level (DAS28-CRP) and the number of edema-positive bones. At least one shared-epitope $H L A-D R B 1^{*} 0405$ allele was found in 36 (45\%) of the 80 patients; this occurred in significantly more edema-positive patients than edema-negative patients.

The authors conclude that their scoring system, particularly in terms of bone edema, determines disease status in patients with early-stage RA. Prospective, follow-up studies are required to confirm these findings.

Original article Tamai M et al. (2007) Bone edema determined by magnetic resonance imaging reflects severe disease status in patients with early-stage rheumatoid arthritis. J Rheumatol 34: 2154-2157

\section{Fibrin is a potential therapeutic target for the treatment of inflammatory disease}

Thrombin mediates fibrin polymerization and is known to be involved in acute and chronic inflammatory processes in vivo. Flick et al. have used various genetically engineered mouse models to study the role of fibrin in the development and progression of arthritis.

In a collagen-induced model of arthritis, genetically engineered lack of fibrin resulted in reduced incidence and severity of disease. In control mice, fibrin deposition was apparent in arthritic knee joints, and the extent of deposition seemed to correlate with the degree of joint pathology. Fibrin was not required for leukocyte trafficking into joints in the early stages of disease, but was shown to support local leukocyte activation and subsequent, secondary, leukocyte infiltration.

The leukocyte-integrin-binding feature of fibrin was required for arthritic progression; although deposits of a mutant form of fibrin lacking the $\alpha_{M} \beta_{2}$-integrin-binding motif could be found in joints of genetically engineered mice immunized with collagen, symptoms of disease were comparable to mice with no fibrin. Expression of tumor necrosis factor, interleukin $1 \beta$ and interleukin 6 was diminished in mice with no fibrin, and in mice expressing only the form unable to bind the leukocyte $\alpha_{M} \beta_{2}$ integrin, compared with control mice. 